\title{
LETTER TO THE EDITOR Reprogramming of Notch1-induced acute lymphoblastic leukemia cells into pluripotent stem cells in mice
}

Blood Cancer Journal (2016) 6, e444; doi:10.1038/bcj.2016.57; published online 8 July 2016

Somatic cells can be reprogrammed into induced pluripotent stem (iPS) cells using the reprogramming factors (Oct4, Sox2, KIf4 and C-Myc, also called OSKM). ${ }^{1}$ Cellular reprogramming and oncogenesis share many common features. The application of the iPS technology in cancers help us better understand the mechanism underlying the initiation and progression of cancer. Therefore, defining the reprogramming potential of cancer cells would provide unique opportunities to reveal epigenetic mechanisms and develop novel therapeutics for cancer. Because the reprogramming efficiency of cancer cells is paradoxically much lower than that of normal cells in general, only some handful types of cancer cells have been explored using the iPS technology. ${ }^{2}$ To date, in the hematopoietic system, Epstein-Barr virus (EBV)transformed lymphoblastoid cell lines, human chronic myeloid leukemia cells, juvenile myelomonocytic leukemia cells and primary murine mixed lineage leukemia-AF9 acute myeloid leukemia cells have been successfully generated into iPS cells. ${ }^{3-6}$ However, whether the primary malignant leukemic T cells can be reprogrammed into the iPS cells is still a mystery.

We first employed OSKM transgenic mice in which OSKM factors can be induced by doxycycline (Dox) and established the T-cell acute lymphoblastic leukemia (T-ALL) mouse model by transfecting the Lineage negative ( $\mathrm{Lin}^{-}$) bone marrow cells from the OSKM mice with a Notch1-green fluorescent protein (GFP) retrovirus (Figure 1a). The mice developed leukemia within 2 months (Supplementary Figure S1A). The moribund mice exhibited a T-ALL phenotype (Supplementary Figures S1B-S1D). The flowchart shows the reprogramming scheme for the T-ALL cells (Figure $1 \mathrm{~b}$ ). GFP ${ }^{+}$leukemia cells were sorted and plated on mouse embryonic fibroblast feeder cells. After the formation of mouse embryonic stem (ES)-like colonies, a single colony was picked up and cultured on feeder cells to produce iPS cell lines (Figure 1c). Overall, the reprogramming efficiency was very low, only approximately $0.005 \pm 0.0005 \%$ (Supplementary Figure S1E). Similarly to our previous study on acute myeloid leukemia cells. ${ }^{4}$ GFP was not expressed in the established leukemia iPS (L-iPS) cells (Figure 1d), indicating that the retroviral vector was silenced in L-iPS cells. The expression of pluripotency markers Oct4, Nanog and SSEA-1 was confirmed by immunofluorescence staining (Figure 1e) and qRT-PCR analyses (Supplementary Figure S2A). Genomic PCR demonstrated the presence of ectopic Notch 1 and immunoglobulin heavy chain rearrangement in all tested L-iPS cells, confirming that L-iPS cells were indeed derived from T-ALL cells (Supplementary Figures S2B and S2C). Moreover, the L-iPS cell lines were predominantly diploid with the normal (40, XY) karyotype (Supplementary Figure S2D).

To further investigate the developmental potential of the L-iPS cells, we performed the teratoma assay in severe combined immunodeficiency (SCID) mice. The teratomas showed all three germ layers (Figures $1 \mathrm{f}-\mathrm{h}$ ). Furthermore, L-iPS cell lines were randomly selected for chimeric assessment, and two L-iPS cell lines generated eight postnatal chimeras, as chimerism reflected by coat color (Figure 1i). Notably, the chimeric mice developed recurrent leukemia within 50 days (Supplementary Figures S2E$\mathrm{S} 2 \mathrm{H})$. Therefore, we were unable to test germ-line transmission using the chimeras. Taken together, we successfully established T-ALL-derived iPS cell lines even at a very low efficiency and further characterized the pluripotency of the L-iPS cells.

Interestingly, during the reprogramming process, we found two types of primary colonies, GFP ${ }^{+}$and $\mathrm{GFP}^{-}$colonies (Figure 2a). Both types of colonies showed typical ES-like morphology. However, only GFP- colonies could be passaged to form iPS cell lines (Figure $2 \mathrm{~b}$ ), indicating an incomplete reprogrammed state of $\mathrm{GFP}^{+}$colonies and a complete reprogrammed state of GFP colonies. The inhibition of Notch has been shown to greatly improve the reprogramming efficiency of both mouse and human cells $s^{6,7}$. The retroviral-expressed Notch 1 in certain leukemia cells was not sufficient to silence. This may explain at least in part why the $\mathrm{GFP}^{+}$colonies could not be fully reprogrammed into iPS cells. Therefore, to understand the differences between the GFP ${ }^{+}$and $\mathrm{GFP}^{-}$colonies, we compared their gene expression by microarray. The gene expression profile of $\mathrm{GFP}^{-}$colonies was much more similar to L-iPS cells than the $\mathrm{GFP}^{+}$colonies (Supplementary Figure S3A). The gene expression trend showed that the expression of pluripotency genes, such as Nanog, Sox2, Oct4 and Sall4, were continuously increased from the leukemia cells to the L-iPS cells (Supplementary Figure S3B).

The gene expression differences between $\mathrm{GFP}^{+}$and $\mathrm{GFP}^{-}$ colonies may provide insights into the potential barriers for reprogramming of T-ALL cells. Gene set enrichment analysis demonstrated that apoptosis, NF-KB, DOT1L, LSD1 and HDAC signature genes were significantly enriched in the $\mathrm{GFP}^{+}$colonies compared with the GFP ${ }^{-}$colonies (Figure $2 \mathrm{c}$ and Supplementary Table S1). As previous studies have reported that NF-KB served as a barrier for reprogramming. ${ }^{8}$ and inhibitors for apoptosis, DOT1L, LSD1 and HDAC were able to improve the reprogramming efficiency. ${ }^{9-12}$ We wanted to know whether these signaling pathways played pivotal roles in T-ALL cell reprogramming. Therefore, we used Z-VAD-FMK, Micheliolide, EPZ004777, Tranylcypromine and Valproic acid (VPA) as inhibitors for apoptosis, NF-KB, DOT1L, LSD1 and HDAC, respectively. To ascertain whether inhibition of these pathways can improve the reprogramming of T-ALL cells, we treated leukemia cells with these inhibitors during reprogramming induction in cultures. Alkaline phosphatase staining revealed slightly higher reprogramming efficiency in individual inhibitor treated group than in dimethylsulphoxide control, except in the VPA-treated group (Figures 2d and e). Notably, we observed more than seven-fold increase in reprogramming efficiency when the cocktail of the five inhibitors was used (Figures $2 \mathrm{~d}$ and e). In consideration that VPA had no effects on T-ALL cells reprogramming efficiency, we then used the cocktail of only four inhibitors without VPA (Figures $2 d$ and e). The result showed that VPA had no contribution to the cocktail. Therefore, the four-inhibitor cocktail suggested a synergistic effect on reprogramming. Taken together, our results indicate that the inhibition of apoptosis, NF-KB, DOT1L and LSD1 can increase the reprogramming efficiency of T-ALL cells.

Reprogramming is a multi-step process involving extensive changes in the transcriptional and epigenetic states. Our study demonstrated that the primary mouse T-ALL cells can also be fully 
a
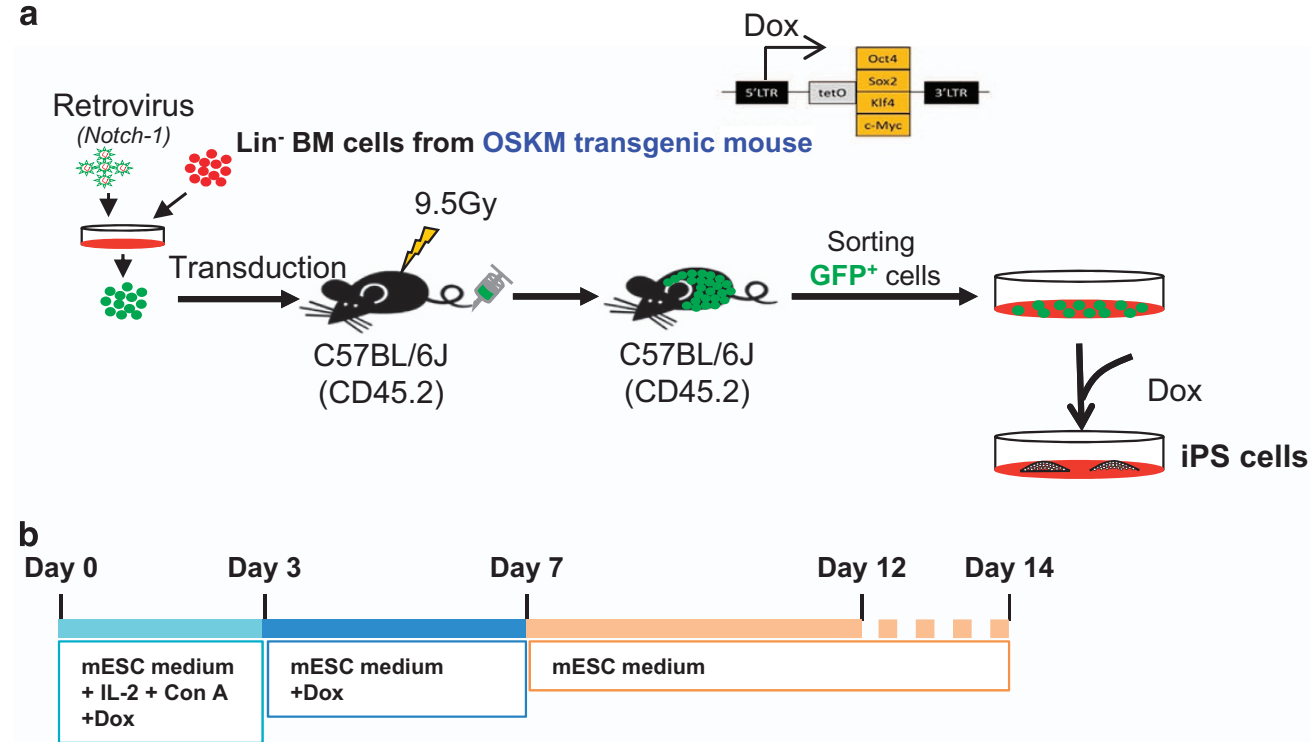

C

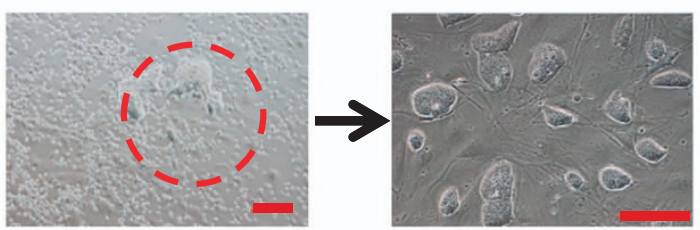

d leukemia cells

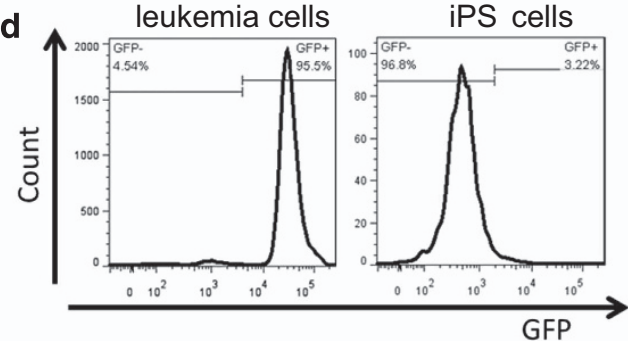

e

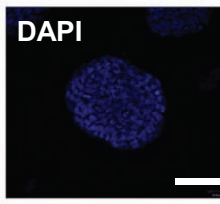

DAPI
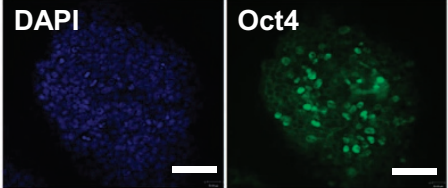

DAPI

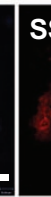

h

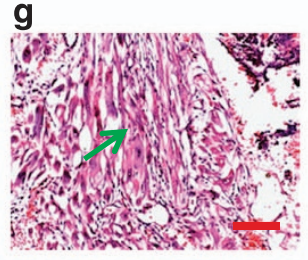

Mesoderm

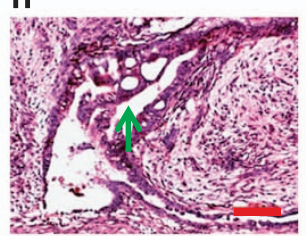

Endoderm
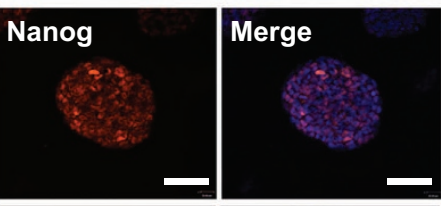

Merge

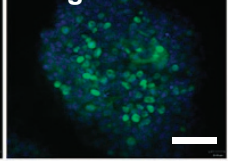

Merge

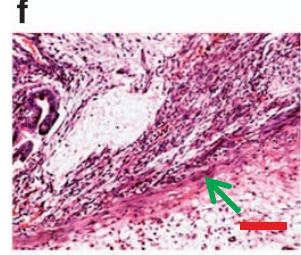

Ectoderm

Figure 1. Reprogramming of T-ALL cells into iPS cells. (a) Experimental scheme for the reprogramming of primary T-ALL cells into iPS cells. The transduced cells were injected into lethally irradiated recipients $(9.5 \mathrm{~Gy})$. (b) Reprogramming procedure for leukemia cells. GFP ${ }^{+}$leukemia cells were sorted and plated on mouse embryonic fibroblast cells at day 0. After 3 days of culture, the medium was changed to ES medium with $2 \mu \mathrm{g} / \mathrm{ml}$ Dox. After ES cell-like colonies appeared at day 7, the medium was replaced with ES medium until the colonies were picked up. (c) Representative morphology of original and passaged colonies of L-iPS cells. Scale bars, $100 \mu \mathrm{m}$. (d) FACS analysis of GFP expression in primary leukemia cells and iPS cells. (e) Immunofluorescence staining showing the expression of pluripotency markers (Nanog, Oct4 and SSEA-1) in L-iPS cells. Scale bars, $60 \mu \mathrm{m}$. (f-h) H\&E staining showing teratoma from L-iPS cells containing all three germ layers. The arrows show the nerve fibers (f), muscular tissues $(\mathbf{g})$ and bronchi (h), respectively. Scale bars, $100 \mu \mathrm{m}$. (i) Chimeric mice generated by blastocyst injection of L-iPS cells. Viable chimeric mice were shown by the coat color. H\&E, hematoxylin and eosin.

reprogrammed into an iPS state and revealed the barriers in the reprogramming process for T-ALL cells. NF-KB was previously showed as a barrier for reprogramming, and DOT1L served as a prominent effector of NF-KB signaling. ${ }^{8}$ Moreover, NF-KB pathway was highly activated in human T-ALL and inhibition of the pathway could significantly restrict the development of Notch1induced T-ALL. ${ }^{13}$ suggesting that the oncogenesis of T-ALL was apparently different from the induced pluripotent reprogramming progress. Furthermore, given the fact that activation of NF-KB pathway was shown to be associated with leukemia stem cells. ${ }^{14}$ forced reprogramming of bulk leukemia cells did not necessarily promote the process toward the leukemia stem cell state. Therefore, further studies are certainly required to fully characterize the relationship between reprogramming and stemness, 
especially in the context of a hematopoietic malignant state. DOT1L inhibition could synergize with Notch inhibition to induce pluripotency at a high efficiency. ${ }^{7}$ Moreover, LSD1 acted as a Notch transcriptional coactivator in the context of the Notch-activation complex. ${ }^{15}$ Therefore, several barriers in our study were associated with chromatin-modifying enzymes, but a

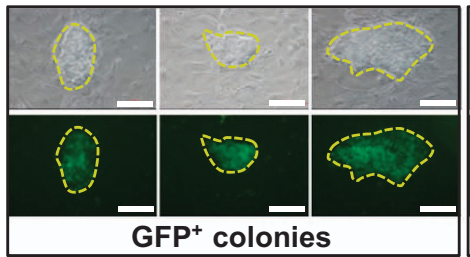

C

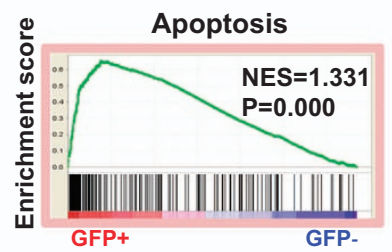

LSD1 up-related genes

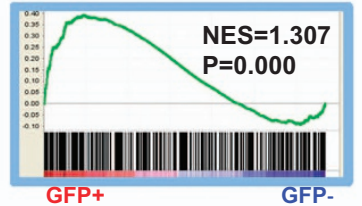

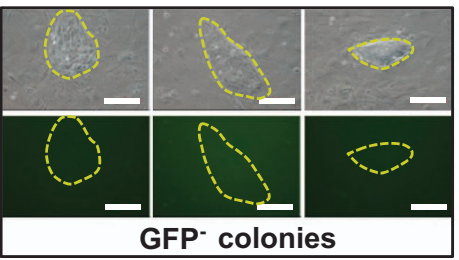

b

\begin{tabular}{|c|c|c|}
\hline Type & $\begin{array}{c}\text { Percentage } \\
\text { of colonies }\end{array}$ & $\begin{array}{c}\text { Percentage of } \\
\text { passage }\end{array}$ \\
\hline GFP $^{+}$ & $47 \%$ & 0 \\
\hline GFP- & $53 \%$ & $85 \%$ \\
\hline
\end{tabular}

DOT1L up-related genes
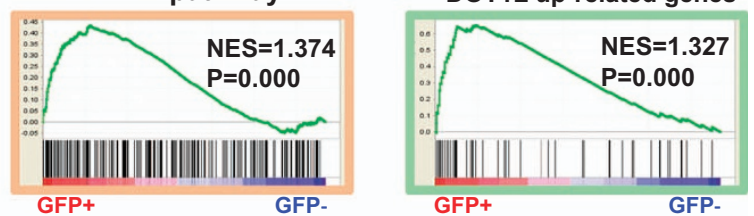

HDAC related genes

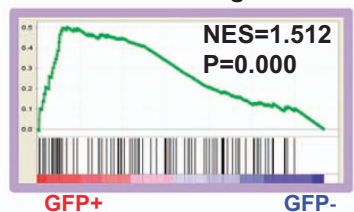

MCL

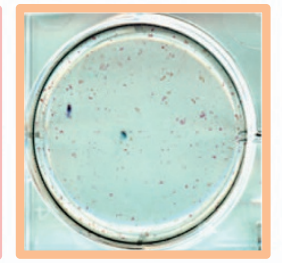

Cocktail

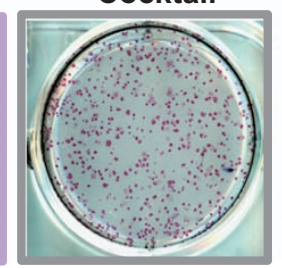

EPZ

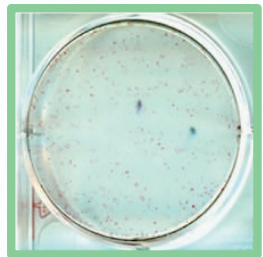

Cocktail (-VPA)

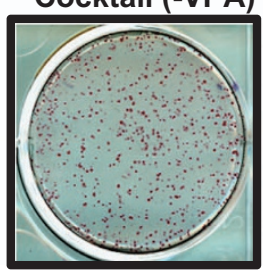

e

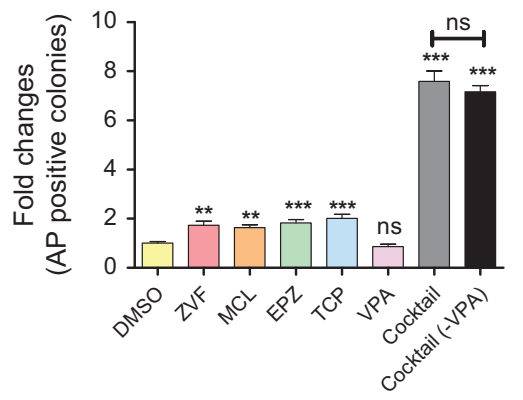

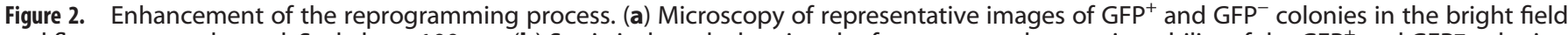
and fluorescence channel. Scale bars, $100 \mu \mathrm{m}$. (b) Statistical result showing the frequency and passaging-ability of the GFP ${ }^{+}$and GFP colonies, $^{-}$ see Figure 2a. (c) Gene set enrichment analysis plots revealing the expression profiles of apoptosis, NF-KB, DOTL1L, LSD1 and HDAC signature genes in $\mathrm{GFP}^{+}$and $\mathrm{GFP}^{-}$colonies. (d) Alkaline phosphatase staining of iPS colonies derived from leukemia cells cultured in systems supplemented with dimethylsulphoxide and $10 \mu \mathrm{m}$ Z-VAD-FMK, $1 \mu \mathrm{m}$ Micheliolide, $10 \mu \mathrm{m}$ EPZ004777, $10 \mu \mathrm{m}$ Tranylcypromine, $500 \mu \mathrm{m}$ VPA, the cocktail and the cocktail without VPA. GFP ${ }^{+}$leukemia cells $\left(1 \times 10^{6}\right.$ cells per well) were plated on feeders in six-well plates in ES medium with Dox. (e) Histograms of relative reprogramming efficiency showing by $\mathrm{AP}^{+}$colonies. $\left(n=6,2\right.$ independent experiments). ${ }^{*} P<0.05,{ }^{* *} P<0.01$, $* * * P<0.001$. Error bars indicate s.e.m. 
their precise mechanisms in reprogramming remain elusive. The relationship between the chromatin modulation and the reprogramming process need to be further studied. In this work, we identified apoptosis, NF-KB, DOT1L and LSD1 pathways as barriers to T-ALL cell reprogramming by showing abortion of reprogramming and enriched their pathways in reprogrammingincompetent colonies compared to reprogramming-competent colonies. More importantly, their inhibitors were able to improve the reprogramming efficiency of T-ALL cells, and the combination increased obviously. Nevertheless, the study shows that the reprogramming of T-ALL cells to pluripotency will provide a more efficient and valuable way for the study of molecular and epigenetic events of T-cell malignancies.

\section{CONFLICT OF INTEREST}

The authors declare no conflict of interest.

\section{ACKNOWLEDGEMENTS}

We thank Dr Shaorong Gao for providing the OSKM transgenic mice. We are grateful to our lab members and collaborators for their assistance with the experiments and in the preparation of the manuscript. This work was supported by grants from the Ministry of Science and Technology of China (2011CB964801, 2012CB966601) and from the National Nature Science Foundation of China (81090411, 81421002, $81430004,91519315,81400077$ and 81300375).

\section{AUTHOR CONTRIBUTIONS}

$\mathrm{HZ}$ performed all the experiments, analyzed the data and wrote the paper. $\mathrm{HC}$ analyzed the data and wrote the paper. $\mathrm{YW}$ and $\mathrm{YZ}$ contributed to all the experiments and data analyses. YL participated in establishing the model. $\mathrm{KL}$ performed the chimera experiments. JX helped with culture mouse embryonic fibroblast cells. SH helped the data analysis. WY conducted research and assisted with manuscript. TZ helped establish the chimeric protocols. TC conceived the study, designed the experiments, interpreted the results and wrote the paper.

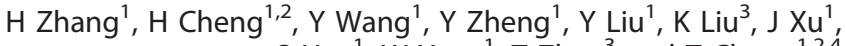 $\mathrm{S} \mathrm{Hao}^{1}, \mathrm{~W}$ Yuan ${ }^{1}, \mathrm{~T}$ Zhao ${ }^{3}$ and T Cheng ${ }^{1,2,4}$}

${ }^{1}$ State Key Laboratory of Experimental Hematology, Institute of Hematology \& Blood Diseases Hospital, Chinese Academy of Medical Sciences \& Peking Union Medical College, Tianjin, China;

${ }^{2}$ Center for Stem Cell Medicine, Chinese Academy of Medical Sciences, Tianjin, China;

${ }^{3}$ State Key Laboratory of Reproductive Biology, Institute of Zoology, Chinese Academy of Sciences, Beijing, China and

${ }^{4}$ Department of Stem Cell and Regenerative Medicine, Peking Union Medical College, Tianjin, China E-mail: chengtao@ihcams.ac.cn

\section{REFERENCES}

1 Takahashi K, Yamanaka S. Induction of pluripotent stem cells from mouse embryonic and adult fibroblast cultures by defined factors. Cell 2006; 126: 663-676.

$2 \mathrm{Kim} \mathrm{JJ}$. Applications of iPSCs in cancer research. Biomark Insights 2015; 10: $125-131$.

3 Kumano K, Arai S, Hosoi M, Taoka K, Takayama N, Otsu M et al. Generation of induced pluripotent stem cells from primary chronic myelogenous leukemia patient samples. Blood 2012; 119: 6234-6242.

4 Liu Y, Cheng H, Gao S, Lu X, He F, Hu L et al. Reprogramming of MLL-AF9 leukemia cells into pluripotent stem cells. Leukemia 2014; 28: 1071-1080.

5 Gandre-Babbe S, Paluru P, Aribeana C, Chou ST, Bresolin S, Lu L et al. Patientderived induced pluripotent stem cells recapitulate hematopoietic abnormalities of juvenile myelomonocytic leukemia. Blood 2013; 121: 4925-4929.

6 Choi SM, Liu H, Chaudhari P, Kim Y, Cheng L, Feng J et al. Reprogramming of EBV-immortalized B-lymphocyte cell lines into induced pluripotent stem cells. Blood 2011; 118: 1801-1805.

7 Ichida JK, Julia TCW, Williams LA, Carter AC, Shi Y, Moura MT et al. Notch inhibition allows oncogene-independent generation of iPS cells. Nat Chem Biol 2014; 10: 632-639.

8 Soria-Valles C, Osorio FG, Gutierrez-Fernandez A, De Los AA, Bueno C, Menendez P et al. NF-kappaB activation impairs somatic cell reprogramming in ageing. Nat Cell Biol 2015; 17: 1004-1013.

9 Kawamura T, Suzuki J, Wang YV, Menendez S, Morera LB, Raya A et al. Linking the p53 tumour suppressor pathway to somatic cell reprogramming. Nature 2009; 460: $1140-1144$.

10 Onder T, Kara N, Cherry A, Sinha AU, Zhu N, Bernt KM et al. Chromatin-modifying enzymes as modulators of reprogramming. Nature 2012; 483: 598-602.

11 Li W, Zhou H, Abujarour R, Zhu S, Young JJ, Lin T et al. Generation of humaninduced pluripotent stem cells in the absence of exogenous Sox2. Stem Cells 2009; 27: 2992-3000.

12 Huangfu D, Maehr R, Guo W, Eijkelenboom A, Snitow M, Chen AE et al. Induction of pluripotent stem cells by defined factors is greatly improved by small-molecule compounds. Nat Biotechnol 2008; 26: 795-797.

13 Vilimas T, Mascarenhas J, Palomero T, Mandal M, Buonamici S, Meng F et al. Targeting the NF-kappaB signaling pathway in Notch1-induced T-cell leukemia. Nat Med 2007; 13: 70-77.

14 Jordan CT. The leukemic stem cell. Best Pract Res Clin Haematol 2007; 20: 13-18.

15 Yatim A, Benne C, Sobhian B, Laurent-Chabalier S, Deas O, Judde J et al. NOTCH1 nuclear interactome reveals key regulators of its transcriptional activity and oncogenic function. Mol Cell 2012; 48: 445-458.

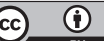

This work is licensed under a Creative Commons Attribution 4.0 International License. The images or other third party material in this article are included in the article's Creative Commons license, unless indicated otherwise in the credit line; if the material is not included under the Creative Commons license, users will need to obtain permission from the license holder to reproduce the material. To view a copy of this license, visit http://creativecommons.org/licenses/ by/4.0/

(c) The Author(s) 2016

Supplementary Information accompanies this paper on Blood Cancer Journal website (http://www.nature.com/bcj) 